\title{
Estenose carotídea acima de $70 \%$ em pacientes no pré-operatório de cirurgia da aorta abdominal: freqüência e fatores de risco
}

\author{
Frequency and risk factors for carotid stenosis above 70\% \\ in patients undergoing abdominal aortic surgery
}

\section{André Ventura Ferreira, Vanessa Prado dos Santos, Roberto Augusto C affaro*}

\section{Resumo}

O bjetivo: Analisar a freqüência e os fatores de risco associados à estenose carotídea acima de $70 \%$ em pacientes que serão submetidos a cirurgias de aorta abdominal.

M aterial e método: Foram analisados 94 pacientes que realizaram ultra-som $\mathrm{D}$ oppler de carótidas no pré-operatório de cirurgias de aorta abdominal entre janeiro de 2000 e janeiro de 2003, pela disciplina de Cirurgia Vascular da Santa Casa de São Paulo.

Resultados: Sessenta e sete pacientes (71\%) eram homens. D entre os 94 pacientes, $42(44,6 \%)$ tinham doença oclusiva aorto-ilíaca, e $52(53,4 \%)$, aneurismas da aorta abdominal (AAA). A análise dos dados mostrou uma prevalência de estenose de carótidas acima de $70 \%$ em $8,33 \%$ dos pacientes com AAA e em $13,51 \%$ dos pacientes com doença oclusiva aorto-ilíaca, diferença esta sem significância estatística $(P=0,5)$. Nos pacientes que apresentavam antecedente de isquemia cerebral - acidente vascular cerebral (AVC) ou ataque isquêmico transitório (AIT) - , houve uma prevalência estatisticamente maior de estenose carotídea entre 70 e $99 \%$. 0 utros fatores de risco para aterosclerose, como sexo masculino, diabetes, hipervensão arterial etabagismo, não foram preditivos da presença de estenose carotídea acima de $70 \%$.

C onclusão: A freqüência de estenose da carótida acima de $70 \%$ em pacientes no pré-operatório de cirurgia de aorta foi de $9,57 \%$, e a presença de antecedente de AVC ou AIT na história foi preditiva de estenose acima de $70 \%$ neste grupo de pacientes.

Palavras-chave: Estenose da carótida, cirurgia da aorta, aterosclerose, aneurismas da aorta abdominal, doença aterosclerótica oclusiva aorto-ilíaca.

A aterosclerose é uma doença sistêmica queacometetodo o organismo ${ }^{1,2}$. Entretanto, pouco éconhecido acerca da coexistência entre a estenose de carótidas e a

\footnotetext{
* Departamento de Cirurgia, Disciplina de Cirurgia Vascular, Faculdade de Ciências Médicas, Santa Casa de São Paulo, São Paulo, SP.

Artigo submetido em 22.11.05, aceito em 20.03.06.
}

J Vasc Br 2006;5(1):17-22.

Copyright $\odot 2006$ by Sociedade Brasileira de Angiologia e de Cirurgia Vascular.

\begin{abstract}
O bjective: To analyze the frequency and risk factors of carotid stenosis above $70 \%$ in patients undergoing abdominal aortic reconstruction.
\end{abstract}

$M$ aterial and method: Ninety-four patients who underwent Doppler ultrasound preoperative screening for abdominal aortic surgery between January 2000 and January 2003 were analyzed by the Vascular Surgery U nit of the Santa C asa of São Paulo (Faculty of M edical Sciences).

Results: Sixty-seven (71\%) patients were male. Of the 94 patients, $42(44.6 \%)$ had aortoiliac occlusive disease and 52 (53.4\%) had abdominal aortic aneurysm. The data analysis showed a prevalence of carotid stenosisabove $70 \%$ in $8.33 \%$ of patients with abdominal aortic aneurysms and $13.51 \%$ in patients with aortoiliac occlusive disease, a difference without statistical significance $(P=0.5)$. T heprevious history of cerebral ischemic symptoms (stroke and transitory ischemic attack) was predictive of the presence of a $70-99 \%$ carotid stenosis. Other risk factors for atherosclerosis, such as being male, having diabetes, hypertension and smoking history, were not predictive of the presence of a carotid stenosis above $70 \%$.

Conclusion: The frequency of carotid stenosis above $70 \%$ in patients undergoing abdominal aortic surgery is $9.57 \%$ and the presence of transitory ischemic attack or stroke in medical history was predictive of stenosis above $70 \%$ in this group of patients.

Key words: Carotid stenosis, aortic surgery, atherosclerosis, abdominal aortic aneurysms, atherosclerotic aortoiliac occlusive disease.

doença aterosclerótica oclusiva aorto-ilíaca ( $D A O A I)$ e aneurisma de aorta abdominal $(A A A)^{1}$. Alguns relatos naliteratura mostram que essa concomitância existeem 20 a $40 \%$ e 17 a $21 \%$ dos casos, respectivamente 3 .

Apesar de raro, no entanto, alguns pacientes submetidos a cirurgias para reconstrução da aorta apresentam como complicação o acidente vascular cerebral isquêmico ( $\mathrm{AVC} \mathrm{C}$ ) ou o ataque isquêmico transitório $(\mathrm{AIT})^{4}$. 
0 objetivo do presente estudo é, portanto, encontrar com quefreqüência ocorre estenose carotídea entre 70 e $99 \%$, ou seja, estenose que exige tratamento cirúrgico aberto ou endovascular, em pacientes que serão submetidos à cirurgia de aorta; se há diferença quando comparamos essa ocorrência entre os portadoresdeD AO AI eAAA; eseexistem fatorespreditivosque indiquem a ocorrência de estenose carotídea superior a $70 \%$, o que justificaria seu tratamento cirúrgico.

\section{M aterial e métodos}

Foram incluídos, em nosso estudo, 94 pacientes, durante o período compreendido entre janeiro de 2000 e janeiro de 2003, com idades entre 42 e 75 anos, em pré-operatório de cirurgia eletiva sobre a aorta abdominal por DAOAI ou AAA, os quais foram acompanhados na disciplina de C irurgia V ascular do $D$ epartamento de C irurgia da I rmandade de M isericórdia da Santa Casa de São Paulo. D entre esses 94 pacientes, $42(44,6 \%)$ se encontravam em programação cirúrgica para reconstrução de aorta por DAOAI, e $52(53,4 \%)$, por AAA.

O s pacientes foram submetidos a exame de ultrasom D oppler colorido de artérias carótidas comuns, externas e internas, no pré-operatório da aorta, no Serviço de D iagnóstico por I magem da Santa C asa de São Paulo, mensurando o grau de estenose da carótida interna.

Analisamos as seguintes variáveis para os 94 pacientes: presença ou não de estenose carotídea entre 70 e $99 \%$, parâmetro este considerado como de tratamento cirúrgico, segundo o estudo N ASCET ${ }^{5}$; antecedentede AVCi ou AIT (isquemia cerebral); presença de diabetes melito (D M ); presença dehipertensão arterial sistêmica (H AS) diagnosticada previamente e em tratamento; presença de tabagismo; sexo.

Comparamos os pacientes divididos em dois grupos, com D AO Al ecom AAA, econjuntamente, em um grupo só, acerca da presença ou não dos parâmetros considerados fatores de risco supramencionados, utilizando-se, para tanto, de análise estatística com o teste exato de Fisher. Consideramos como significativos os valores de $\mathrm{P} \leq 0,05$.

\section{Resultados}

D entre as variáveis estudadas, encontramos que 22 (23\%) doentes apresentavam D M , 68 (72\%) H AS, 64 (68\%) tabagismo, e $11(11,7 \%)$ antecedente de isque- mia cerebral, AVCi e/ ou AIT . Estenose carotídea entre 70 e $99 \%$ esteve presente em 9 (9,57\%) doentes.

Q uando comparamos a ocorrência de estenose carotídea entre 70 e $99 \%$ no grupo de pacientes que apresenta AAA e no grupo que apresenta DAOAI, encontramos valores iguais a 8,33 e $13,51 \%$, respectivamente, com um valor de $P=0,5$, ou seja, diferença de prevalência não estatisticamente significante entre os dois grupos.

Ao analisarmos a presença de estenose de carótidas entre 70 e99\% com aocorrência prévia ou não deAV Ci e/ou AIT , obtivemos diferença estatisticamentesignificativa em todos os grupos comparados. $\mathrm{N}$ o grupo de pacientes com AAA, 100\% dos doentes com estenose entre 70 e $99 \%$ apresentavam antecedente de isquemia cerebral, enquanto que apenas $6,25 \%$ dos doentes sem estenose carotídea significativa apresentavam esse antecedente, com valor deP =0,0001 (Tabela 1). N o grupo de pacientes com DAOAI, 60\% dos pacientes com estenose carotídea entre 70 e $99 \%$ apresentavam antecedente de isquemia cerebral, enquanto que apenas 2,7\% dos pacientes sem estenose carotídea significativa apresentavam o mesmo antecedente, com $\mathrm{P}=0,0033$ (T abela 2). Q uando analisados os grupos juntamente (D AO I eAAA), encontramosque $77,8 \%$ dospacientes com estenose carotídea entre 70 e $99 \%$ apresentavam antecedente de AVCi e/ ou AIT, enquanto que 4,7\% dos pacientes sem estenosesignificativa apresentavam 0 mesmo antecedente, com P =0,000001 (T abela 3).

D entreas outras variáveis anal isadas, não encontramos diferença estatisticamente significativa em nenhum dos outros fatores (Tabela 4). No grupo de doentes com D M , 13,6\% apresentavam estenose carotídea entre 70 e $99 \%$, e $86,4 \%$ não a tinham, enquanto que, no grupo de pacientes não-D M , 8,3\% apresentavam estenose entre 70 e $99 \%$, e $91,7 \%$ não ( $P=0,432)$.

Tabela 1 - Estenose carotídea superior a 70\% em aneurismas da aorta abdominal

\begin{tabular}{lcc}
\hline $\begin{array}{l}\text { Aneurismas da } \\
\text { aorta abdominal }\end{array}$ & $\begin{array}{c}\text { Estenose } \\
>\mathbf{7 0 \%}\end{array}$ & $\begin{array}{c}\text { Estenose } \\
<70 \%\end{array}$ \\
\hline AVC/AIT & $4(100 \%)$ & $3(6,25 \%)$ \\
Sem AVC/AIT & 0 & $45(93,75 \%)$ \\
Total & $4(100 \%)$ & $48(100 \%)$
\end{tabular}

$\mathrm{AIT}=$ ataque isquêmico transitório; $\mathrm{AVC}=$ acidente vascular cerebral $P=0,0001$. 
Em relação à H AS, no grupo de pacientes hipertensos, $10,3 \%$ apresentavam estenose entre 70 e $99 \%$ e $89,7 \%$ não; já no grupo dos não-H AS, 7,7\% cursavam com estenose entre 70 e $99 \%$, e 92,3\% não ( $P=1)$. D entre

T abela 2 - Estenose carotídea superior a 70\% em doença aterosclerótica oclusiva aorto-ilíaca

\begin{tabular}{lcc}
\hline $\begin{array}{l}\text { D oença aterosclerótica } \\
\text { oclusiva aorto-ilíaca }\end{array}$ & $\begin{array}{c}\text { Estenose } \\
\mathbf{>} \mathbf{7 0} \%\end{array}$ & $\begin{array}{c}\text { Estenose } \\
<\mathbf{7 0} \%\end{array}$ \\
\hline AVC/AIT & $3(60 \%)$ & $1(2,7 \%)$ \\
Sem AVC/AIT & $2(40 \%)$ & $36(97,3 \%)$ \\
Total & $5(100 \%)$ & $37(100 \%)$ \\
\hline
\end{tabular}

$\mathrm{AIT}=$ ataque isquêmico transitório; $\mathrm{AVC}=$ acidente vascular cerebral. $P=0,0033$.

T abela 3 - Estenosecarotídeasuperior a $70 \%$ em isquemia cerebral

\begin{tabular}{lcc}
\hline D AOAI + AAA & $\begin{array}{c}\text { Estenose } \\
\mathbf{> 7 0} \%\end{array}$ & $\begin{array}{c}\text { Estenose } \\
<\mathbf{7 0} \%\end{array}$ \\
\hline AVC/AIT & $7(77,8 \%)$ & $4(4,7 \%)$ \\
Sem AVC/AIT & $2(22,2 \%)$ & $81(95,3 \%)$ \\
Total & $9(100 \%)$ & $85(100 \%)$ \\
\hline
\end{tabular}

$\mathrm{AAA}=$ aneurisma da aorta abdominal; $\mathrm{AIT}=$ ataque isquêmico transitório $A V C=$ acidente vascular cerebral; DAOAI = doença aterosclerótica oclusiva aorto-ilíaca.

$P=0,000001$

T abela 4 - Fatores de risco em estenose carotídea superior a $70 \%$

\begin{tabular}{lccc}
\hline D AOAI + AAA & $\begin{array}{c}\text { Estenose } \\
>\mathbf{7 0} \%\end{array}$ & $\begin{array}{c}\text { Estenose } \\
<\mathbf{7 0} \%\end{array}$ & $\mathbf{P}$ \\
\hline D M & $3(13,6 \%)$ & $19(86,4 \%)$ & 0,432 \\
N ão-D M & $6(8,3 \%)$ & $66(91,7 \%)$ & \\
H AS & $7(10,3 \%)$ & $61(89,7 \%)$ & 1 \\
N ão-H AS & $2(7,7 \%)$ & $24(92,3 \%)$ & \\
T abagista & $7(9,7 \%)$ & $65(90,3 \%)$ & 0,71 \\
N ão-tabagista & $2(9,1 \%)$ & $20(90,9 \%)$ & \\
M asculino & $6(9,0 \%)$ & $61(91 \%)$ & 0,71 \\
Feminino & $3(11,1 \%)$ & $24(88,9 \%)$ &
\end{tabular}

AAA = aneurisma da aorta abdominal; DAOAI = doença aterosclerótica oclusiva aorto-ilíaca; DM = diabetes melito; HAS = hipertensão arterial sistêmica. os pacientes tabagistas, 9,7\% apresentavam estenose entre 70 e $99 \%$, e $90,3 \%$ não, enquanto que, no grupo dos não-tabagistas, 9,1\% tinham estenose entre $70 \mathrm{e}$ $99 \%$, e $90,9 \%$ não $(P=0,71)$. Q uando comparados com relação ao sexo, 9\% dos pacientes do sexo masculino apresentavam estenose entre 70 e99\%, e91\% não, enquanto $11,1 \%$ dos doentes do sexo feminino cursavam com estenose entre 70 e $99 \%$, e 88,9\% não tinham estenose acima de $70 \%(P=0,71)$.

$Q$ uando analisamos conjuntamente a presença de qualquer dos fatores de risco - DM, HAS ou tabagismo - e o grau de estenose, também não encontramos diferença estatisticamente significativa, com $\mathrm{P}=1,0$. N o grupo de doentes que apresentava pelo menos um fator de risco, 9,8\% apresentavam estenose carotídea entre 70 e $99 \%$, enquanto que, no grupo sem nenhum fator de risco, composto por apenas dois pacientes, nenhum pacienteapresentou esse grau de estenose ( $T$ abela 5 ).

Tabela 5 - Fatores de risco versus grau de estenose

\begin{tabular}{lcc}
\hline AAA + D AOAI & $\begin{array}{c}\text { Estenose } \\
>\mathbf{7 0 \%}\end{array}$ & $\begin{array}{c}\text { Estenose } \\
<70 \%\end{array}$ \\
\hline $\begin{array}{l}\text { DM e/ou H AS } \\
\text { elou T ab }\end{array}$ & $9(9,8 \%)$ & $83(90,2 \%)$ \\
$\begin{array}{l}\text { N ão-D M e/ou } \\
\text { não-H AS e/ou não-T ab }\end{array}$ & 0 & $2(100 \%)$
\end{tabular}

$\mathrm{AAA}=$ aneurisma da aorta abdominal; $\mathrm{DAOAI}=$ doença aterosclerótica oclusiva aorto-ilíaca; DM = diabetes melito; HAS = hipertensão arterial sistêmica; $T a b=$ tabagismo.

$P=1,0$.

\section{D iscussão}

Após o estudo N ASCET ${ }^{5}$ de 1991, ficou estabelecido que pacientes com estenose carotídea entre 70 e $99 \%$ e sintomáticos, com antecedente recente de AVCi e/ou AIT, beneficiam-se de endarterectomia de carótidas. Esse procedimento foi inicialmente proposto em 1954, justamente como uma maneira de prevenir a isquemia cerebral decorrente do desprendimento de um êmbolo a partir de uma placa ateromatosa que provoca estenose carotídea significativa $^{6}$. Segundo alguns autores, essa é a segunda principal causa de isquemia cerebral, ficando atrás somente de fonte cardíaca de êmbolos 2,7 . 
Sabe-se que a concomitância de estenose carotídea entre 70 e $99 \%$ e AAA ou D AO AI que necessitem de tratamento cirúrgico é variável, tendo sido relatadas incidências de 8 a 9\% segundo alguns autores, e, quando consideradas estenoses carotídeas acima de $50 \%$, essa prevalência pode chegar de 24 a 40\% $1,8-10$. C heng et al. ${ }^{9}$ demonstraram que pacientes com doença vascular periférica apresentam, em 23,7\% dos casos, estenose carotídea significativa. Por sua vez, Kang et al. ${ }^{11}$, em 1999, relataram em seu estudo que pacientes com estenoses carotídeas superiores a $50 \%$ apresentam maior risco de serem portadores de AAA; no entanto, poucos autores estratificam as estenoses, obtendo separadamente as que são de indicação cirúrgica, segundo N ASCET ${ }^{5}$ e ACAS ${ }^{12}$, como nosso estudo.

Em nosso estudo, encontramos que a prevalência de estenose carotídea cirúrgica (entre 70 e 99\%) nos pacientes com AAA foi igual a 8,33\%, enquanto que, nos pacientes com D AOAI, foi igual a 13,51\%. Estes dados foram semelhantes aos encontrados na literatura e corroboram a assertiva de quea doença aterosclerótica é uma doença sistêmica, e, na nossa opinião, tal prevalência justifica a triagem desses pacientes para doença carotídea.

L evando-se em conta esses dados e sabendo-se que o risco de ocorrência de instabilidade hemodinâmica durante a cirurgia de um paciente submetido à reconstrução da aorta é alto ${ }^{13}$, podendo provocar, portanto, isquemia cerebral nos doentes com estenose carotídea significativa, faz-senecessário discutir a importância da realização de exames de imagem que possam diagnosticar estenose carotídea significativa no pré-operatório dessas operações. 0 risco de ocorrência de acidente vascular cerebral (AVC) hemorrágico nesse grupo de pacientes pode ser considerado baixo ${ }^{14}$; entretanto, 0 mesmo não pode ser dito acerca do risco de ocorrência deAVCi e/ ou AIT. N o nosso H ospital, após a triagem com ultra-som D oppler, o paciente com estenose carotídea acima de $70 \%$ é submetido à arteriografia e ao tratamento cirúrgico da artéria carótida, antes da cirurgia da aorta abdominal.

Axelrod et al. ${ }^{3}$, em 2002, mostraram-se contrários à realização de exames diagnósticos nos casos de pacientes com AAA sem queixas clínicas referentesà existência de estenose carotídea passível de tratamento cirúrgico, sendo que esses exames só deveriam ser realizados nos pacientes que relatassem sintomas sugestivos da presença de estenose carotídea significativa. C ahan et al., em $1999^{1}$, por sua vez, consideraram que a realização do estudo de imagem para o diagnóstico de estenose carotídea entre 70 e $99 \%$ deve ser realizado para todos os doentes em programação cirúrgica para reconstrução da aorta, independentemente da sintomatologia exibida, por apresentar uma boa relação custo/benefício e por diminuir a incidência de isquemia cerebral no pósoperatório ${ }^{1,4,12}$. Entretanto, M acchi et al., em 2002 ${ }^{15}$, concluíram que, devido à variação individual do calibre da aorta e de suas ramificações, a estenose carotídea mensurada percentualmente, quando analisada isoladamente, não pode ser considerada como um critério confiável para a escolha do tratamento, mas a presença de uma artéria carótida interna de pequeno diâmetro bilateral pode ser considerada como um fator de risco isoladamente. $\mathrm{N}$ ós, por outro lado, não encontramos outros estudos na literatura que emitissem opinião semelhante, sendo que a mensuração da estenose carotídea em percentagem éuniversal menteaceita como um critério diagnóstico e, após o estudo N ASCET ${ }^{5}$, terapêutico.

C ahan et al. ${ }^{1}$ demonstraram, em sua casuística, que a estenose carotídea significativa foi mais freqüentemente encontrada entre os pacientes com DAOAI do que entre os com AAA ( 10 versus $4 \% ; P=0,046$ ). $\mathrm{N} 0$ nosso trabalho, quando comparamos a freqüência de estenose carotídea entre 70 e $99 \%$ nos dois diferentes grupos (13,51 versus8,33\%), não obtivemossignificância estatística. Talvez dando seqüência a esse estudo, como é nosso objetivo, e aumentando nossa amostra, essa diferença se mostre significante.

N osso estudo encontrou queum dado dehistória do paciente (antecedente de isquemia cerebral) pode servir como um preditor para a ocorrência de estenose carotídea entre 70 e 99\%, auxiliando-nos, portanto, a realizar uma investigação detal hada em todos os pacientes sintomáticos do ponto de vista cerebral, em pré-operatório de cirurgia de aorta. N o entanto, também obtivemos pacientes assintomáticos quanto a antecedente de isquemia cerebral que apresentavam estenose carotídea entre 70 e 99\%, merecendo também tratamento cirúrgico segundo critérios do $\mathrm{ACAS}^{12}$, o que, ao nosso ver, justifica a triagem de tais pacientes.

Bechtel et al., em $2000^{13}$, demonstraram, em sua casuística, que aproximadamente 13\% dos doentes apresentavam antecedentes positivos para isquemia cerebral, dentre os quais apenas $57,7 \%$ apresentavam estenose carotídea significativa. Em nossa casuística, por sua vez, encontramos estenose carotídea entre 70 e 
99\% em $63 \%$ dos pacientes com antecedente de isquemia cerebral ( $T$ abela 3 ), sendo que, diferentemente do que é mostrado na literatura, esse dado de história do doente, por si só, mostrou-se um fator preditivo de estenose carotídea significativo. A pesar de os relatos da literatura não terem feito essa correlação, alguns trabaIhos corroboram nossos achados, mostrando uma incidência de estenose carotídea superior a 50\% nos pacientes com antecedente de isquemia cerebral variável de 34,5 a $57,7 \% 9,13,16$.

A presença ou não de fatores de risco como H AS, D M , tabagismo ou sexo, analisados deformaisolada ou em conjunto, não se mostrou preditora de estenose carotídea entre 70 e $99 \%$ em nosso estudo. Contudo, vale ressaltar que apenas dois de nossos doentes não apresentavam quaisquer fatores de risco para doença aterosclerótica (T abelas 4 e5). Fukuda et al. ${ }^{16}$ demonstraram que a D M representa um fator de risco isolado para estenose carotídea superior a 50\%. Cheng et al. ${ }^{9}$ mostraram que o tabagismo, o sexo masculino ea idade são fatores de risco significativos para ocorrência de estenose carotídea entre 70 e 99\%, diferentemente do que encontramos em nosso estudo. Kallikazaros et al. ${ }^{2}$, por outro lado, demonstraram que a presença de idade avançada, H AS e D M são fatores de risco significativos para ocorrência de placas de aterosclerose nas artérias carótidas, fato estequeencontra respaldo fisiopatológico. Entretanto, 0 autor em questão não relata 0 grau de estenose carotídea percentualmente, não sendo seus resultados comparáveis, portanto, aosnossos. Cahan et al. ${ }^{1}$ demonstraram em seu estudo que apenas a idade avançada, dentre as variáveis por eles analisadas, mostrou-se como um fator de risco significativo para a redução do grau de estenose carotídea. Kuukasjärvi et al. ${ }^{17}$ relataram ainda que a isquemia de extremidades é um fator de risco significativo para ocorrência de estenose carotídea superior a 60\%; entretanto, este dado não foi passível decomparação, em decorrência da falta de outros trabal hos analisando a mesma variável.

\section{C onclusões}

Concluímos, conforme os nossos dados, que a estenose carotídea de indicação cirúrgica (entre 70 e $99 \%$ ) esteve presente em $9,57 \%$ dos candidatos à cirurgia de aorta abdominal, não sendo encontrada diferença estatisticamente significativa entre os grupos D AO Al eAAA. A estenosecarotídea entre 70 e99\% foi significativamente mais prevalente em pacientes com antecedente de isquemia cerebral (AVC ou AIT).

\section{Agradecimento}

Agradecemos ao N úcleo de Apoio à Publicação da Faculdade de C iências M édicas da Santa C asa de São Paulo (N AP-SC) o suporte técnico-científico à publicação deste manuscrito.

\section{R eferências}

1. Cahan M A, Killewich $L A$, Kolodner $L$, et al. The prevalence of carotid artery stenosis in patients undergoing aortic reconstruction. Am J Surg. 1999;178(3):194-6.

2. Kallikazaros IE, T sioufis CP, Stefanadis CI, Pitsavos CE, T outouzasPK. C losed relation between carotid and ascending aortic atherosclerosis in cardiac patients. Circulation. 2000;102(19 suppl 3):III263-8.

3. Axelrod DA, Diwan A, Stanley JC, et al. Cost of routine screening for carotid and lower extremity occlusive disease in patients with abdominal aortic aneurysms. J Vasc Surg. 2002;35(4):754-8.

4. Bower T C, M errell SW , C herry K J J r., et al. Advanced carotid disease in patients requiring aortic reconstruction. Am J Surg. 1993;166(2):146-51.

5. N orth American Symptomatic C arotid Endarterctomy T rial Collaborators. Beneficial effect of carotid endarterectomy in symptomatic patients with high-grade carotid stenosis. $\mathrm{N}$ Engl J M ed. 1991;325(7):445-53.

6. Eastcott $\mathrm{HH}$, Pickering GW, Rob CG. Reconstruction of internal carotid artery in a patient with intermittent attacks of hemiplegia. Lancet. 1954;267(6846):994-6.

7. Autret A, Pourcelot L, Saudeau D, M archal C, Bertrand P, de Boisvilliers S. Stroke risk in patients with carotid stenosis. Lancet. 1987;1(8538):888-90.

8. M arek J, M ills JL, H arvich J, C ui H, Fujitani RM . U tility of routine carotid duplex screening in patients who have claudication. J V asc Surg. 1996;24(4):572-7.

9. Cheng SW, W u LL, Lau H , T ing AC, W ong J. Prevalence of significant carotid stenosisin C hinese patients with peripheral and coronary artery disease. Aust N Z J Surg. 1999;69(1):44-7.

10. Deville $C$, Kerdi S, Madonna $F$, de la Renaudiere DF, Labrousse $L$. Infrarenal abdominal aortic aneurysm repair: detection and treatment of associated carotid and coronary lesions. Ann Vasc Surg. 1997;11(5):467-72.

11. Kang SS, Littooy FN, G upta SR, et al. H igher prevalence of abdominal aortic aneurysms in patients with carotid stenosis but without diabetes. Surgery. 1999;126(4):687-91.

12. Executive Committee for the Asymptomatic Carotid Atherosclerosis Study. Endarterectomy for asymptomatic carotid artery stenosis. JAM A. 1995;273(18):1421-8.

13. Bechtel JF, Bartels C, Hopstein S, Horsch S. Carotid endarterectomy prior to major abdominal aortic surgery. J Cardiovasc Surg. 2000;41(2):269-73.

14. Cheung RT, Eliasziw $M$, M eldrum $H E$, Fox AJ, Barnett $H$ J. Risk, types, and severity of intracranial hemorrhagein patients with symptomatic carotid artery stenosis. Stroke. 2003;34(8):1847-51. 
15. M acchi C, Lova RM, M iniati $B$, et al. Is the percentage of stenosis of the internal carotid artery a reliable measure of the risk of ischemic stroke? A morphometric study by duplex ultrasound of aortic arch branches in 500 normal adults. J Cardiovasc Surg. 2002;43(1):71-6.

16. Fukuda I, Gomi S, W atanabe K, Seita J. Carotid and aortic screening for coronary artery bypass grafting. Ann Thorac Surg. 2000;70(6):2034-9.

17. Kuukasjarvi P, Kaira P, Riekkinen H, Salenius JP. Carotid stenosis and abdominal aortic aneurysm at the follow-up examination in patientstreated for acute extremity ischaemia. J Cardiovasc Surg (Torino). 2000;41(2):275-9.
Correspondência:

$\checkmark$ anessa Prado dos Santos

OM B, Cirurgia Vascular

Rua C esário M ota Jr., 112

CEP 01221-900 - São Paulo, SP

Tel.: (11) 2176.7273

Fax: (11) 2176.7273

E-mail: vansanbr@hotmail.com

O conteúdo do J Vasc Br está disponível em português e em inglês

no site do Jornal Vascular Brasileiro em

www.jvascbr.com.br 